\title{
Analogue Hawking radiation and quantum soliton evaporation in a superconducting circuit
}

\author{
Zehua Tian ${ }^{1,2,3,4, a}$, Jiangfeng Du ${ }^{1,2,3, b}$ \\ ${ }^{1}$ Hefei National Laboratory for Physical Sciences at the Microscale and Department of Modern Physics, University of Science and Technology of \\ China, Hefei 230026, China \\ ${ }^{2}$ CAS Key Laboratory of Microscale Magnetic Resonance, University of Science and Technology of China, Hefei 230026, China \\ ${ }^{3}$ Synergetic Innovation Center of Quantum Information and Quantum Physics, University of Science and Technology of China, Hefei 230026, \\ China \\ ${ }^{4}$ Key Laboratory for Research in Galaxies and Cosmology, Chinese Academy of Science, 96 JinZhai Road, Hefei 230026, Anhui, China
}

Received: 6 July 2019 / Accepted: 26 November 2019 / Published online: 9 December 2019

(C) The Author(s) 2019

\begin{abstract}
Hawking radiation is one of the most intriguing and elusive predictions of quantum field theory in curved spacetime. Previous works simulating Hawking radiation have been mostly based on Unruh's scenario, where the propagation of quantum field in classical gravitational background is mimicked. Here, guided by the duality between black holes in Jackiw-Teitelboim (JT) dilaton gravity and solitons in sine-Gordon (SG) field theory, we propose the use of a superconducting circuit for investigating analogue Hawking radiation. $1+1$ dimensional black holes can be realized as solitons of the SG equation of superconducting phase. It is found despite the absence of field theoretic dynamical modes, the analogue Hawking radiation is emitted in terms of the quantum soliton evaporation as a result of quantum perturbation of the black hole metric. Our theoretical proposal could not only facilitate the observation of relativistic quantum effects in lab, but also contribute to experimentally exploring the quantum mechanics of solitons, especially to the deep relationship between such mechanics and black hole physics.
\end{abstract}

\section{Introduction}

Hawking radiation is particle creation stimulated by quantum vacuum fluctuations in the black hole background [1,2], revealing the deep link between gravity and thermodynamics [3], as well as quantum mechanics. Observation of Hawking radiation could not only facilitate the exploration of the still-unknown laws of quantum gravity, but also plays a very important role in addressing the black hole information loss

\footnotetext{
a e-mail: tianzh@ustc.edu.cn

be-mail: djf@ustc.edu.cn
}

paradox $[4,5]$. However, demonstrating Hawking radiation is probably extremely difficult through direct astrophysical black hole observations, since typical stellar size black holes are cold and are far away from us. To render the relevant physics accessible to an experimental investigation, Unruh [6] first pointed out that sound waves in a convergent fluid flow behaves the same as quantum field in a classical gravitational field. He thus predicted that a thermal spectrum of sound waves should be given out from the sonic horizon in transsonic fluid flow, provided that the incoming acoustic modes are in vacuum state. This is the analogue Hawking radiation. Note that initial thermal noise could affect the thermal nature of radiation spectrum, and the relevant issues have been investigated by D. E. Bruschi et al. [7]. Recently, a lot of "analogous gravity" experiments have been proposed to observe the analogue Hawking radiation [8-17]; see Ref. [18] for an extensive review and a comprehensive list of references.

On the other hand, there is a duality between black holes in JT dilaton gravity and solitons in SG field theory [19]. Unlike the previous derivation of Hawking radiation, in this theory the JT black holes emit the thermal radiation in terms of quantum soliton evaporation as a result of the perturbation of black hole metric [20]. Besides, they still exhibit the usual thermodynamic properties even without the field theoretic dynamical modes [21]. Therefore, this theory might provide us a new perspective to shed light on the field theory origin of black holes and the dynamical source of black hole entropy $[19,21]$.

Previous papers focused either on the duality between black holes in JT dilaton gravity and solitons in SG field theory, or only on the physics of SG field theory in some special systems, such as DNA-promoter dynamics [22]. There 
are few proposals involving the experiments with fantastic controllability and scalability to demonstrate these physics, especially to further explore their deep connection. In this paper, we theoretically provide an experimental proposal-a superconducting electrical circuit configuration-to reconsider the relevant elements together. Particularly, we pay our attention to the realization of $1+1$ dimensional black holes as solitons of the SG equation of superconducting phase. The analogue Hawking radiation is demonstrated in terms of the quantum soliton evaporation. Our theoretical study fills the gap of analogue gravity, which is based on the duality between solitons in SG field theory and black holes in JT dilaton gravity theory. Furthermore, it provides a new experimental tool to manifest this duality and learn the physics of SG field theory.

Note that our scenario is based on the well established circuit quantum electrodynamics (cQED) technology [23,24]. It is of the fantastic controllability and scalability, and thus could offer a natural arena for testing fundamentals of quantum mechanics and implementing quantum field theory concepts [25]. Moreover, our proposed setup is quite similar to the superconducting coplanar waveguide (CPW) [26,27] and the direct-current superconducting quantum interference devices (SQUID) array transmission line [28,29] (see more details below), which have already been constructed experimentally with parameters near those required in our proposed setup to observe the Hawking effect [28-31]. Our theoretical proposal therefore can in principle be achievable within the current cQED technology.

Our paper is constructed as following: in Sect. 2 we simply review the duality between black holes in JT dilaton gravity and solitons in SG field theory. In Sect. 3 we introduce our proposed setup and study how to simulate the SG equation with superconducting phase. Sections 4 and 5 are respectively devoted to the investigation of Hawking radiation in SG field theory and the possible experimental implementation of our proposal. Conclusions and discussions are given in Sect. 6.

\section{Sine-Gordon soliton black hole}

It has long been known that the solutions of the SG equation, $\partial_{t}^{2} \phi-\partial_{x}^{2} \phi+m^{2} \sin \phi=0$, determine Riemannian geometries with constant negative curvature $-2 m^{2}$ [32]. The line-element corresponding to this manifold is, $d s^{2}=\sin ^{2}(\phi / 2) d t^{2}+\cos ^{2}(\phi / 2) d x^{2}$, where the angle $\phi$ describes the embedding of the manifold into a three dimensional Euclidean space [32]. Its corresponding Lorentzian geometries with line-element $d s^{2}=-\sin ^{2}(\phi / 2) d \tau^{2}+$ $\cos ^{2}(\phi / 2) d \xi^{2}$ have negative constant curvature if and only if

$\frac{\partial^{2} \phi}{\partial \tau^{2}}+\frac{\partial^{2} \phi}{\partial \xi^{2}}=\sin \phi$, where a Wick rotation $t \rightarrow$ it following Ref. [19] and the new variables $\tau=m t$ and $\xi=m x$ have been used. Note that for the JT gravity with action $I_{\mathrm{JT}}[\psi, g]=$ $\frac{1}{2 G} \int_{M_{2}} d x^{2} \sqrt{|g|} \psi\left(R+2 m^{2}\right)$ in this Lorentzian geometries, Eq. (1) actually provides a sufficient condition to determine that the equation of motion of Ricci scalar satisfies $R=-2 m^{2}$, i.e., that spacetime $M_{2}$ has a Lorentzian metric $g_{\mu \nu}$ with constant negative curvature $-2 m^{2}$. Therefore, according to this interesting relation the duality between black holes in JT dilaton gravity and solitons in SG field theory has been built [19]. It allows us to realize the constant negative curvature two dimensional black holes as solitons of the SG equation.

As an example, we shall now demonstrate that the 1soliton solution of the elliptic SG equation (1) determines a black hole metric. The 1-soliton solution of the elliptic SG equation can be written as

$\phi(\tau, \xi)=4 \arctan \exp \left[ \pm \gamma\left(\xi-\beta_{s} \tau\right)\right]$

where $\gamma=\left(1+\beta_{s}^{2}\right)^{-1 / 2}$, and the constant $\beta_{s}$ is a "spectral parameter" satisfying $0<\beta_{s}<1$. The solution with the "+" sign in the exponent is the 1-soliton solution, while the opposite sign is the anti-soliton solution. Substituting this solution into the Lorentzian line-element above, following Ref. [19] we can obtain the Schwarzschild form,

$d s^{2}=\left(\beta_{s}^{2}-r^{2}\right) d \mathcal{T}^{2}-\left(\beta_{s}^{2}-r^{2}\right)^{-1} d r^{2}$.

Here the definitions $d \mathcal{T}=d \tau-\beta_{s} \frac{\tanh ^{2} \rho}{\gamma\left(\operatorname{sech}^{2} \rho-\beta_{s}^{2} \tanh ^{2} \rho\right)} d \rho$ and $r=\frac{1}{\gamma} \operatorname{sech} \rho \geq 0$ with $\rho=\gamma\left(\xi-\beta_{s} \tau\right)$ have been used. This metric describes a JT black hole with event horizon at $r_{\mathrm{H}}=\beta_{s}$. It is actually a dimensionally truncated three dimensional BTZ black hole [33-35].

\section{Sine-Gordon equation in superconducting circuit}

We theoretically propose a setup shown in Fig. 1 to realize JT black holes as solitons of SG euquation. Note that our configuration is similar to the CPW in Refs. [26,27], however, in our configuration each capacitor is parallel with an identical SQUID. It is the added SQUIDs that can provide a nonlinear potential to the Lagrangian of fluxes in the CPW, which plays a very crucial role in the simulation of black hole.

In this work, each SQUID consists of two identical parallel Josephson junctions (JJs). Besides, the geometric size of SQUID loop is assumed to be small enough such that its selfinductance is negligible compared to its kinetic inductance. In this case, each SQUID can be referred to as an effective $\mathrm{JJ}$ with a junction capacitance $C_{\mathrm{J}}$ and a tunable Josephson energy $E_{\mathrm{J}}\left(\Phi_{\text {ext }}^{\mathrm{J}}\right)=2 E_{\mathrm{J}} \cos \left(\pi \frac{\Phi_{\text {ext }}^{\mathrm{J}}}{\Phi_{0}}\right)[27]$. Here $\Phi_{0}=h / 2 e$ is the magnetic flux quantum, $E_{\mathrm{J}}=\frac{\Phi_{0} I_{\mathrm{c}}}{2 \pi}$ is the Josephson 


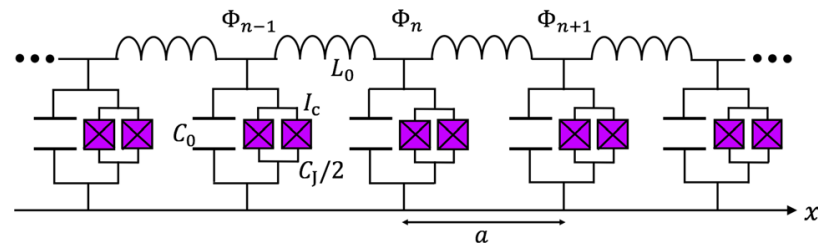

Fig. 1 Circuit diagram for a coplanar waveguide-like transmission line. The inductance for each inductor and the capacitance for each capacitor are assumed to be $L_{0}$ and $C_{0}$, respectively. Each SQUID element consists of two identical tunnel Josephson junctions with critical current $I_{\mathrm{c}}$ and capacitance $\frac{1}{2} C_{\mathrm{J}}$. The length of all the cells is the constant $a$. The circuit is characterized by the dynamical fluxes $\Phi_{n}$

energy, and $\Phi_{\text {ext }}^{\mathrm{J}}=B A_{\mathrm{S}}$ is the flux dropping through the SQUID loop with the effective area $A_{\mathrm{S}}$ and the applied magnetic field $B$.

With the quantum-network theory [36], the Lagrangian corresponding to our circuit configuration reads

$$
\begin{aligned}
\mathscr{L}= & \sum_{n=1}^{N}\left[\frac{1}{2} C_{0}\left(\dot{\Phi}_{n}\right)^{2}-\frac{\left(\Phi_{n+1}-\Phi_{n}\right)^{2}}{2 L_{0}}+\frac{1}{2} C_{\mathrm{J}}\left(\dot{\Phi}_{n}\right)^{2}\right. \\
& \left.+E_{\mathrm{J}}\left(\Phi_{\mathrm{ext}}^{\mathrm{J}}\right) \cos \left(2 \pi \frac{\Phi_{n}}{\Phi_{0}}\right)\right]
\end{aligned}
$$

where $\Phi_{n}$ is the dynamical fluxes of the $n$-th node. We restrict ourselves to the macroscopic SQUID junctions in the phase regime, i.e., when the Josephson energy is so big compared to the charging energy, $E_{\mathrm{J}}\left(\Phi_{\mathrm{ext}}^{\mathrm{J}}\right) \gg(2 e)^{2} / 2 C_{\mathrm{J}}$, and oscillations in the phase across the SQUID could satisfy $\frac{\Phi_{n}}{\Phi_{0}} \ll 1$. The small amplitude condition allows us to study the Lagrangian above by linearizing the Josephson cosine terms. In this regard, let us note we have expanded the cosine terms in (3) up to the second order in $\Phi_{n}^{2}$ to investigate the analog cosmological particle creation [37]. However, here we will learn the whole nonlinear terms without any approximation. It is these cosine terms that produce a sine term for the Klien-Gordon equation of the superconducting phase (shown in (4)), which plays a key role in the analog of black hole. In addition, we assume that the wavelength $\lambda$ for the flux is much longer than the dimensions of a single unit cell of the chain, i.e., $a / \lambda \ll 1$. Under this assumption the above Lagrangian (3) can be considered in the continuum approximation, i.e., $\Phi_{n}-\Phi_{n-1} \approx a \frac{\partial \Phi}{\partial x}+o\left(a^{2}\right)$ to the first order of $(a / \lambda)$, written as $\mathscr{L}=\frac{C}{2} \int d x\left[\left(\frac{\partial \Phi}{\partial t}\right)^{2}-\frac{a^{2}}{L_{0} C}\left(\frac{\partial \Phi}{\partial x}\right)^{2}+\right.$ $\left.\frac{E_{\mathrm{J}}\left(\Phi_{\mathrm{ext}}^{\mathrm{J}}\right)}{C} \cos \left(\frac{2 \pi \Phi}{\Phi_{0}}\right)\right]$, with $C=C_{0}+C_{\mathrm{J}}$. Through variation with respect to $\phi=\frac{2 \pi}{\Phi_{0}} \Phi$ and its derivative, we find the superconducting phase $\phi$ satisfies $1+1$ dimensional SG equation, $\frac{\partial^{2} \phi}{\partial t^{2}}-c^{2} \frac{\partial^{2} \phi}{\partial x^{2}}+m^{2} \sin \phi=0$.

Here $c=a / \sqrt{L_{0} C}$ is the velocity of propagation, which in practice is well below the vacuum speed of light $c_{0}$. And $m=\sqrt{4 \pi^{2} E_{\mathrm{J}}\left(\Phi_{\mathrm{ext}}^{\mathrm{J}}\right) / C \Phi_{0}^{2}}$ can be considered as the effective mass. For simplification, hereafter we will assume $c=1$ similar to the conventional natural units.

As discussed above, the solutions of the SG equation (4) determine Riemannian geometries with constant negative curvature $-2 m^{2}$. Adopting coordinates transformation $\tau=m t, \xi=m x$, and $t \rightarrow$ it [19], one can realize the JT black holes as solitons of SG equation in our setup. Thus, it allows us to understand the mechanics of solitons and especially the duality between JT gravity and SG soliton from the perspective of experiment.

\section{Hawking radiation}

Due to the vacuum fluctuation near black hole horizon, virtual particle pairs are constantly created. The negative-energy particles fall into the black hole, reducing the black hole energy, while the particles with positive energy can go far away to the infinite, known as the Hawking radiation. The radiation spectrum is proportional to the particle occupation number in thermal equilibrium, but subject to different statistical distributions depending on what kinds of emitted particles.

For a quantum scalar field in the background of black hole with the metric shown in Eq. (2), we can solve its equation of motion and calculate the spontaneous Hawking radiation with the quantum field theory in curved spacetime [38,39]. It is found the thermal Hawking radiation spectrum is

$$
\left\langle\hat{N}_{\Omega}\right\rangle=\left\langle 0_{\mathrm{K}}\left|\hat{b}_{\Omega}^{\dagger} \hat{b}_{\Omega}\right| 0_{\mathrm{K}}\right\rangle=\left[\exp \left(\frac{2 \pi \Omega}{\beta_{s}}\right)-1\right]^{-1} .
$$

Here $\hat{b}_{\Omega}$ is the annihilation operator for Boulware vacuum, which is singular at the horizon and measured by observers remaining at a constant distance from the black hole. $\left|0_{\mathrm{K}}\right\rangle$ is the Kruskal vacuum that is regular on the horizon, and corresponds to true physical vacuum in the presence of the black hole [39]. The thermal radiation means that seen from the remote observer, the Kruskal vacuum is not vacuum anymore. That is to say, particles are emitted from black hole.

In our proposal, we can obtain the analogue Hawking radiation in the SG field theory by analyzing the weak perturbation of field [40], i.e., by assuming $\phi \simeq \phi_{s}+\phi_{1}$. Here $\phi_{s}$ describes the classical solution to Eq. (1), and $\phi_{1} \ll \phi_{s}$ is the perturbation which satisfies, $\left[\partial_{\tau}^{2}+\partial_{\xi}^{2}\right] \phi_{1}-\cos \left(\phi_{s}\right) \phi_{1}=0$, to the first order of $\phi_{1}$. Following Ref. [20], we can obtain the same result as that shown in (5). Let us note that for the soliton black hole case, the analogue Hawking radiation 
actually behaves as quantum soliton evaporation, which has been obtained even without the interaction with a massless scalar field. It means that JT black holes exhibit the usual thermodynamic properties, including black hole entropy, despite the absence of field theoretic dynamical modes in the theory. Therefore, the deep relation between JT black holes and SG solitons might shed light on the field theory origin of black holes and the dynamical source of black hole entropy.

Let us note that our proposal actually performs in the Eculidean spacetime. In this case, the propagator of quantum field is of the same characteristic of thermal Green function $[41,42]$, i.e., it is periodic with the period being the inverse of temperature of thermal bath. We can rewrite the Schwarzschild metric in Eq. (2) in the Kruskal coordinates,

$d s^{2}=\left(\beta_{s}+r\right)^{2}\left(d T^{2}-d R^{2}\right)$,

where $T=\beta_{s}^{-1}\left(\frac{\beta_{s}-r}{\beta_{s}+r}\right)^{1 / 2} \sinh \left(\beta_{s} \mathcal{T}\right)$ and $R=-\beta_{s}^{-1}$ $\left(\frac{\beta_{s}-r}{\beta_{s}+r}\right)^{1 / 2} \cosh \left(\beta_{s} \mathcal{T}\right)$. With the Wick rotation $\mathcal{T} \rightarrow-i \mathcal{T}^{\prime}$, the analytic continuation of time to imaginary axis is given by $i T=\beta_{s}^{-1}\left(\frac{\beta_{s}-r}{\beta_{s}+r}\right)^{1 / 2} \sin \left(\beta_{s} \mathcal{T}^{\prime}\right)$ and $R=-\beta_{s}^{-1}\left(\frac{\beta_{s}-r}{\beta_{s}+r}\right)^{1 / 2}$ $\cos \left(\beta_{s} \mathcal{T}^{\prime}\right)$. Then the Lorentzian Kruskal metric becomes the Euclidean one, $d s^{2}=-\left(\beta_{s}+r\right)^{2}\left(d T^{2}+d R^{2}\right)$. The imaginary time $\mathcal{T}^{\prime}$ is a periodic coordinate with period $T_{p}=2 \pi / \beta_{s}$, as well as any continuous functions defined on this manifold. Therefore, the propagator of quantum field for the Lorentzian metric case, $G(r, \mathcal{T})$, is also a periodic function of $\mathcal{T}^{\prime}$ with period $2 \pi / \beta_{s}$, provided that it is defined as the analytic continuation of propagator for Euclidean case $[41,42]$. Note that this behavior is characteristic of what are known as "thermal Green's functions", i.e., $G_{\text {th }}(r, \mathcal{T})=G_{\text {th }}\left(r, \mathcal{T}+i T_{p}\right)$. Thus, from the perspective of the observer it will seem as if he were in a bath of blackbody radiation at the temperature $T_{\mathrm{H}}=1 / T_{p}=\beta_{s} / 2 \pi$.

The Hawking temperature $T_{\mathrm{H}}=\beta_{s} / 2 \pi$ depends on the velocity of soliton, $\beta_{s}$. This dependence of the Hawking radiations on the translation velocity is peculiar of soliton dynamics [43], and it is related to the structure of the spectral parameter in the inverse scattering transform $[44,45]$. In addition, the elliptic SG soliton moves with the velocity $\beta_{s}$, and thus the frequency $\Omega$ seen by an observer at rest with respect to the soliton should contain a Doppler shift [20]. As a consequence of that, the Hawking temperature in the laboratory frame reads

$T_{\mathrm{H}}=\frac{\beta_{s}}{2 \pi} \sqrt{\frac{1-\beta_{s}}{1+\beta_{s}}}$,

which can be reduced to $T_{\mathrm{H}} \simeq \frac{\beta_{S}}{2 \pi}\left(1-\beta_{S}\right)$ in the small $\beta_{S}$ limit.

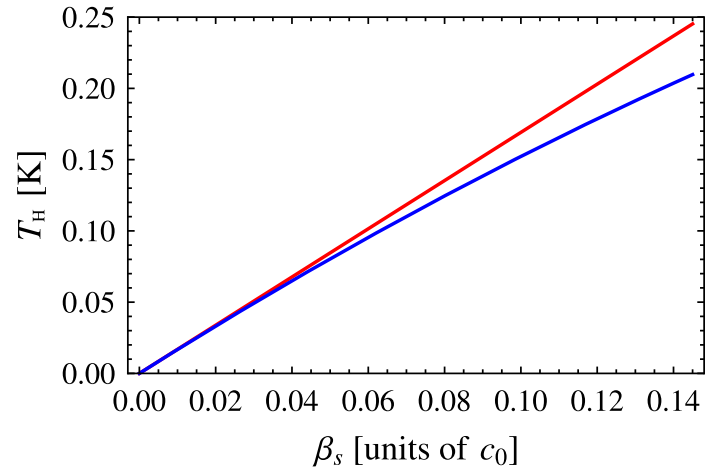

(a)

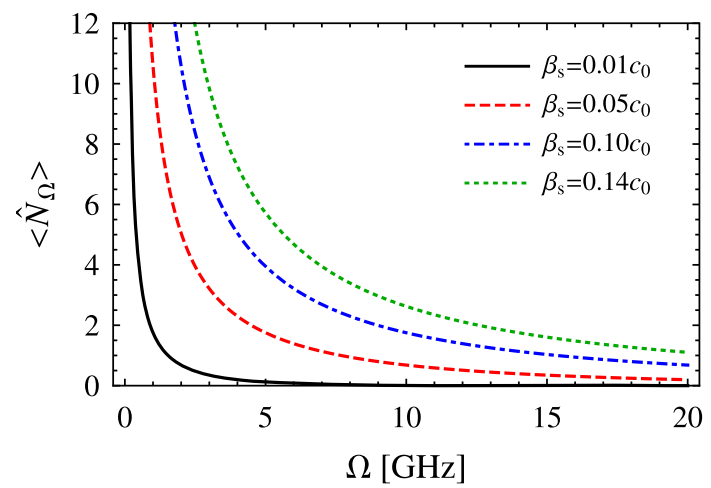

(b)

Fig. 2 a The temperature of analogue Hawking radiation observed in comoving frame (red solid line) and in laboratory frame (blue solid line); b the radiation spectrum of Eq. (5) as a function of particle frequency seen from the comoving frame. Note that the radiation spectrum seen from the laboratory, which is not shown, just has very slightly quantitive difference

\section{Experimental implementation}

To realize the proposed experiment, we import a signal $\phi_{S}$ plus a linear weak perturbation $\phi_{1} \ll \phi_{s}$ into our setup. The signal $\phi_{S}$ behaves as SG soliton and plays the role of black hole, while $\phi_{1}$ can be considered as a quantum perturbation of the black hole metric and induce the analogue Hawking radiation that we aim to observe [20]. The soliton velocity $\beta_{s}$ can be set by preparation of the signal and must not be higher than the unbiased transmission line propagation velocity $c$. Therefore, the Hawking radiation should satisfy $T_{\mathrm{H}} \leq c / 2 \pi$ in the comoving frame, and the radiation power is given by $[10,46,47], d E / d \mathcal{T}=\frac{\pi}{12 \hbar}\left(k_{b} T_{\mathrm{H}}\right)^{2}$. In order to observe the quantum fluctuation, i.e., the analogue Hawking radiation, a frequency-tunable, single-shot photon detection at the end of the transmission line should be prepared. For example, we may choose a superconducting phase qubit as the detector and detect the microwave photon based on the recently proposed technologies [48-51]. As discussed above, in our proposal we consider the relevant physics of analogue gravity in Eculidean space, which is related to the Lorentzian 
one through the Wick rotation. Actually, the propagator of the perturbation $\phi_{1}$, which equivalently plays the role of quantum field in the analogue gravity, is periodic with period $T_{p}=2 \pi / \beta_{s}=1 / T_{H}$. The period is exactly the inverse of expected Hawking temperature. Therefore, the period of the propagator of $\phi_{1}$ can be measured to characterize the analogue Hawking effect.

In the black hole evaporation process, each radiation particle is actually entangled with a negative-energy partner particle, which falls into the black hole and hence reduces the black hole energy [52]. This entanglement verifies the quantum natures of the Hawking radiation. It plays a very important role to evidence that the detected thermal particles are from the emission of black hole, rather than other irrelevant processes, such as ambient emission. In experiment, the coincidence detection could give us an access to such entanglement. Usually, correlation functions or some inequalities [11,13,30,53-57] are used to qualitatively measure the quantum correlations of generated pairs. Note that correlations functions of the quantum SG model have been computed recently [58].

We will choose the relevant parameters for each element similar to Refs. [10,26-31] and estimate the analogue Hawking radiation in our configuration. For the $\mathrm{JJ}$, we assume its critical current and effective junction capacitance are respectively $I_{\mathrm{c}}=2 \mu \mathrm{A}$ and $C_{\mathrm{J}}=1.2 \mathrm{fF}$, and the plasma frequency is thus $\omega_{s}=2 \pi \sqrt{E_{\mathrm{J}} / \Phi_{0}^{2} C_{\mathrm{J}}} \simeq 2.25 \times 10^{12} \mathrm{~Hz}$. Besides, the capacitance to ground is chosen as $C_{0}=0.8 \mathrm{fF}$, the inductance and the length of the single unit cell of our setup are respectively assumed to be $L_{0}=0.01 \mathrm{nH}$ and $a=6 \mu \mathrm{m}$. In this case, the propagation velocity is $c \simeq 0.14 c_{0}$ where $c_{0}$ is the velocity of light in the vacuum. In Fig. 2, we plot the Hawking temperature and the spectrum of Hawking radiation. It is shown that the effective temperature is proportional to the velocity of the soliton, and the number of created particle decreases as the increase of the particle frequency. Let us note that the temperature could be as high as a few $\mathrm{mK}$, which can be a factor of 10 larger than the ambient temperature set by a dilution refrigerator. Therefore, this effect should be visible above the background noise.

Let us see how the unavoidable input thermal noise affects the detection of analogue Hawking radiation. We assume the temperature of the initial thermal noise is $T_{\mathrm{N}}$, then we can obtain the radiation spectrum,

$\left\langle\hat{N}_{\Omega}\right\rangle_{\mathrm{T}_{N}}=\frac{1}{2}\left[\operatorname{coth}\left(\frac{\pi \Omega}{\beta_{S}}\right) \operatorname{coth}\left(\frac{\Omega}{2 T_{\mathrm{N}}}\right)-1\right]$.

Note that this radiation spectrum reduces to the initial thermal spectrum for $T_{\mathrm{H}}=\beta_{s} / 2 \pi=0$, while it goes back to the familiar case in Eq. (5) when starting from the initial vacuum, i.e., $T_{\mathrm{N}}=0$ case. Therefore, the radiation spectrum is thermal only provided that the initial input state of quan-

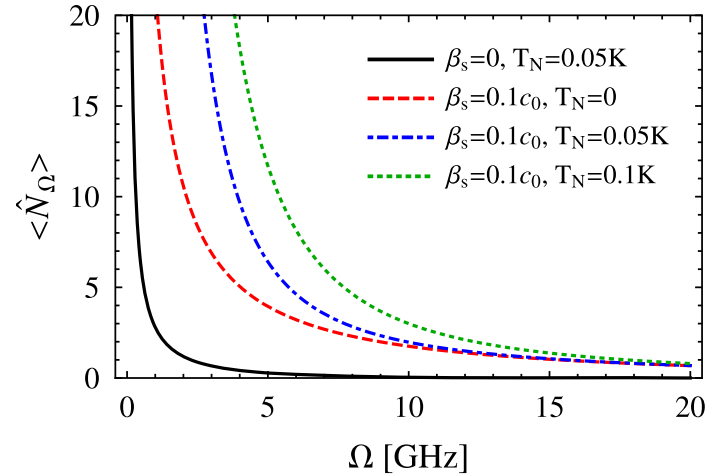

(a)

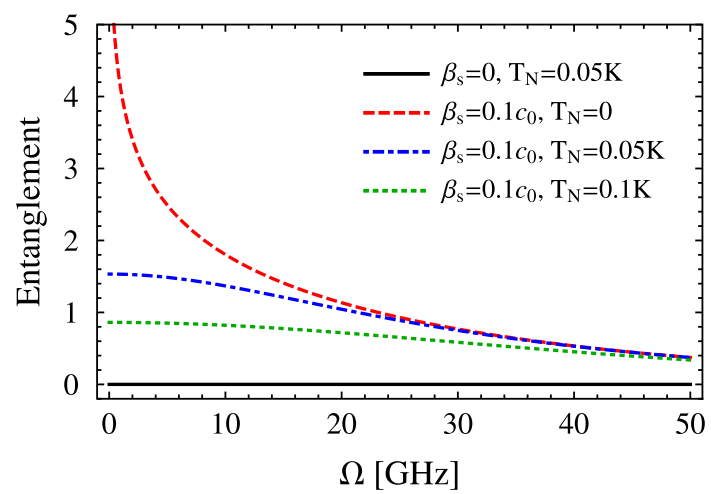

(b)

Fig. 3 a The radiation spectrum of Eq. (8) as a function of particle frequency seen from the comoving frame; $\mathbf{b}$ the entanglement of created particles in the presence of thermal noise as a function of particle frequency seen from the comoving frame

tum field modes is vacuum. In Fig. 3, we plot the analogue Hawking radiation spectrum and the entanglement of created particles in the presence of thermal noise. It is shown that in the presence of thermal noise the radiation spectrum deviates from the thermal case shown in Eq. (5), especially for low energy modes. Furthermore, non-zero temperature suppresses the entanglement of created particles. The entanglement is the criterion to judge whether the detected thermal particles are from the emission of black hole, or from other ambient emission, therefore, the input thermal noise might make the detection of analogue Hawking radiation more difficult. To effectively demonstrate the analogue Hawking radiation in the presence of thermal noise, we need to suppress the initial thermal noise as much as possible, thus keep the radiation spectrum thermal as expected in theory.

The relevant parameters and pulse shapes above were chosen as an example that our setup is theoretically feasible, which should not be considered as the only available configuration. In fact, it is possible to improve and optimize these values in terms of both performance and fabrication of this proposal. On the other hand, properly engineering the transmission line could effectively reduce the background noise 
from the unwanted coupling and hence make the detection of analogue Hawking radiation more effective.

\section{Conclusions and discussions}

In summary, we have provided a recipe to build up a quantum simulator of black hole physics based on superconducting circuit. In our proposed setup, the superconducting phase satisfies the SG equation. Therefore, due to the duality between black holes in JT dilaton gravity and solitons in SG field theory, the solutions of the SG equation can be used to realize the constant curvature two dimensional black holes. Our theoretical results showed that the proposed device works in the quantum region and allows us to observe the analogue Hawking radiation in terms of quantum soliton evaporation.

Let us note that Nation et al. [10] first proposed the use of a superconducting transmission line formed from an array of direct-current SQUID for investigating analogue Hawking radiation. In their scenario, an additional conducting line is required to produce a spacetime varying external flux bias. This external flux can induce a spacetime changing velocity of propagation in the transmission line, thus leading to an effective metric with a horizon. The current pulse dispersion in the bias line is unavoidable and thus results in a decrease in Hawking temperature. Besides, the capacitive coupling to the bias line can spuriously generate photons, which are noise and may make the detection of Hawking radiation more difficult. In contrast, our setup here is similar to the proposed configuration in Ref. [10], but without needing the additional bias line that provides an external flux necessary to modify the SQUID array propagation velocity. Thus, in our scenario fewer dissipation and fewer noise will be introduced in the detection of analogue Hawking radiation.

It is speculated that the black hole entropy may be given by the number of ways of preparing quantum SG states with fixed total energy and soliton number $Q=1[19,59]$. The proposed scenario thus might help us understand the physics of black hole entropy. In addition, our analysis can be extended to a more general case, i.e., constructing SG coordinates for a black hole with $\mathrm{N}$-soliton [59], and cosmological analog [60]. It therefore facilitates the analogue gravity and understanding of quantum mechanics of solitons, as well as the deep relation between dilaton gravity and SG field theory. It is interesting to point out that the effective mass $m$ in Eq. (4) actually can be adjusted by means of a suitable strongly inhomogeneous external magnetic flux bias along a waveguide-like transmission line, and thus is timeand space-dependent. Due to this property, our current setup allows us to investigate a diverse range of areas of physics, such as a nonuniform Josephson junction and DNA-promoter dynamics, which could be described by the SG model with a variable mass [61].
Acknowledgements We thank Jiliang Jing, Yifu Cai, and Ya Wang for the inspired discussions and valuable suggestions. This work was supported by the National Key R\&D Program of China (Grant No. 2018YFA0306600), the CAS (Grants No. GJJSTD20170001 and No. QYZDY-SSW-SLH004), and Anhui Initiative in Quantum Information Technologies (Grant No. AHY050000). Tian was supported by the National Natural Science Foundation of China under Grant Nos. 11905218 , and the CAS Key Laboratory for Research in Galaxies and Cosmology, Chinese Academy of Science (No. 18010203).

Data Availability Statement This manuscript has no associated data or the data will not be deposited. [Authors' comment: All data generated or analyzed during this study are included in this article.]

Open Access This article is licensed under a Creative Commons Attribution 4.0 International License, which permits use, sharing, adaptation, distribution and reproduction in any medium or format, as long as you give appropriate credit to the original author(s) and the source, provide a link to the Creative Commons licence, and indicate if changes were made. The images or other third party material in this article are included in the article's Creative Commons licence, unless indicated otherwise in a credit line to the material. If material is not included in the article's Creative Commons licence and your intended use is not permitted by statutory regulation or exceeds the permitted use, you will need to obtain permission directly from the copyright holder. To view a copy of this licence, visit http://creativecomm ons.org/licenses/by/4.0/.

Funded by SCOAP . $^{3}$

\section{References}

1. S. W. Hawking, Nature 248, 30 EP (1974). https://doi.org/10.1038/ 248030a0

2. S.W. Hawking, Communications in Mathematical Physics 43, 199 (1975), ISSN 1432-0916. https://doi.org/10.1007/BF02345020

3. J.D. Bekenstein, Phys. Rev. D 7, 2333 (1973). https://doi.org/10. 1103/PhysRevD.7.2333

4. F. Wilczek, in International symposium on black holes, membranes, wormholes and superstrings woodlands, Texas, January 16-18, 1992 (1993), pp. 1-21. arXiv:hep-th/9302096

5. P. Chen, G. Mourou, Phys. Rev. Lett. 118, 045001 (2017). https:// doi.org/10.1103/PhysRevLett.118.045001

6. W.G. Unruh, Phys. Rev. Lett. 46, 1351 (1981). https://doi.org/10. 1103/PhysRevLett.46.1351

7. D.E. Bruschi, N. Friis, I. Fuentes, S. Weinfurtner, N. J. Phys. 15, 113016 (2013). URL http://stacks.iop.org/1367-2630/15/i=11/ $a=113016$

8. L.J. Garay, J.R. Anglin, J.I. Cirac, P. Zoller, Phys. Rev. Lett. 85, 4643 (2000). https://doi.org/10.1103/PhysRevLett.85.4643

9. L.J. Garay, J.R. Anglin, J.I. Cirac, P. Zoller, Phys. Rev. A 63, 023611 (2001). https://doi.org/10.1103/PhysRevA.63.023611

10. P.D. Nation, M.P. Blencowe, A.J. Rimberg, E. Buks, Phys. Rev. Lett. 103, 087004 (2009). https://doi.org/10.1103/PhysRevLett. 103.087004

11. D. Boiron, A. Fabbri, P.-E. Larré, N. Pavloff, C.I. Westbrook, P. Ziń, Phys. Rev. Lett. 115, 025301 (2015). https://doi.org/10.1103/ PhysRevLett.115.025301

12. J. Steinhauer, Nat. Phys. 10, 864 EP (2014). https://doi.org/10. 1038/nphys 3104

13. J. Steinhauer, Nat. Phys. 12, 959 EP (2016). https://doi.org/10. 1038/nphys 3863

14. O. Lahav, A. Itah, A. Blumkin, C. Gordon, S. Rinott, A. Zayats, J. Steinhauer, Phys. Rev. Lett. 105, 240401 (2010). https://doi.org/ 10.1103/PhysRevLett.105.240401 
15. B. Horstmann, B. Reznik, S. Fagnocchi, J.I. Cirac, Phys. Rev. Lett. 104, 250403 (2010). https://doi.org/10.1103/PhysRevLett. 104.250403

16. B. Horstmann, R. Schützhold, B. Reznik, S. Fagnocchi, J.I. Cirac, N. J. Phys. 13, 045008 (2011). http://stacks.iop.org/1367-2630/13/ $\mathrm{i}=4 / \mathrm{a}=045008$

17. A. Roldán-Molina, A.S. Nunez, R.A. Duine, Phys. Rev. Lett. 118, 061301 (2017). https://doi.org/10.1103/PhysRevLett.118.061301

18. C. Barceló, S. Liberati, M. Visser, Living Rev. Relat. 14, 3 (2011), ISSN 1433-8351. https://doi.org/10.12942/lrr-2011-3

19. J. Gegenberg, G. Kunstatter, Phys. Lett. B 413, 274 (1997), ISSN 0370-2693. http://www.sciencedirect.com/science/article/ pii/S0370269397011180

20. L.D.M. Villari, G. Marcucci, M.C. Braidotti, C. Conti, J. Phys. Commun. 2, 055016 (2018). http://stacks.iop.org/2399-6528/2/ $\mathrm{i}=5 / \mathrm{a}=055016$

21. J.P.S. Lemos, Phys. Rev. D 54, 6206 (1996). https://doi.org/10. 1103/PhysRevD.54.6206

22. M. Salerno, Phys. Rev. A 44, 5292 (1991). https://doi.org/10.1103/ PhysRevA.44.5292

23. A. Wallraff, D.I. Schuster, A. Blais, L. Frunzio, R.S. Huang, J. Majer, S. Kumar, S.M. Girvin, R.J. Schoelkopf, Nature 431, 162 EP (2004). https://doi.org/10.1038/nature02851

24. X. Gu, A.F. Kockum, A. Miranowicz, Y. xi Liu, F. Nori, Physics Reports 718-719, 1 (2017), ISSN 0370-1573, microwave photonics with superconducting quantum circuits. http://www.sciencedirect. com/science/article/pii/S0370157317303290

25. P.D. Nation, J.R. Johansson, M.P. Blencowe, F. Nori, Rev. Mod. Phys. 84, 1 (2012). https://doi.org/10.1103/RevModPhys.84.1

26. J.R. Johansson, G. Johansson, C.M. Wilson, F. Nori, Phys. Rev. A 82, 052509 (2010). https://doi.org/10.1103/PhysRevA.82.052509

27. J.R. Johansson, G. Johansson, C.M. Wilson, F. Nori, Phys. Rev. Lett. 103, 147003 (2009). https://doi.org/10.1103/PhysRevLett. 103.147003

28. M.A. Castellanos-Beltran, K.W. Lehnert, Appl. Phys. Lett. 91, 083509 (2007). arXiv:0706.2373

29. M.A. Castellanos-Beltran, K.D. Irwin, G.C. Hilton, L.R. Vale, K.W. Lehnert, Nat. Phys. 4, 929 EP (2008). https://doi.org/10. 1038/nphys 1090

30. C.M. Wilson, G. Johansson, A. Pourkabirian, M. Simoen, J.R. Johansson, T. Duty, F. Nori, P. Delsing, Nature 479, 376 EP (2011). https://doi.org/10.1038/nature10561

31. P. Lähteenmäki, G.S. Paraoanu, J. Hassel, P.J. Hakonen, Proc. Natl. Acad. Sci. 110, 4234 (2013), ISSN 00278424. http://www.pnas.org/content/110/11/4234.full.pdf. http:// www.pnas.org/content/110/11/4234

32. R.K. Bullough, P.J. Caudrey, SOLITONS (Springer-verl.(1980) (Topics In Current Physics, 17), Berlin, Germany, 1980)

33. M. Bañados, C. Teitelboim, J. Zanelli, Phys. Rev. Lett. 69, 1849 (1992). https://doi.org/10.1103/PhysRevLett.69.1849

34. M. Bañados, M. Henneaux, C. Teitelboim, J. Zanelli, Phys. Rev. D 48, 1506 (1993). https://doi.org/10.1103/PhysRevD.48.1506

35. M. Bañados, Phys. Rev. D 57, 1068 (1998). https://doi.org/10. 1103/PhysRevD.57.1068

36. B. Yurke, J.S. Denker, Phys. Rev. A 29, 1419 (1984). https://doi. org/10.1103/PhysRevA.29.1419

37. Z. Tian, J. Jing, A. Dragan, Phys. Rev. D 95, 125003 (2017). https:// doi.org/10.1103/PhysRevD.95.125003
38. N.D. Birrell, P.C.W. Davies, Quantum Fields in Curved Space, Cambridge Monographs on Mathematical Physics (Cambridge Univ. Press, Cambridge, UK, 1984). ISBN 0521278589, 9780521278584 , 9780521278584. http://www.cambridge.org/mw/academic/ subjects/physics/theoretical-physics-and-mathematical-physics/ quantum-fields-curved-space format $=\mathrm{PB}$

39. V.F. Mukhanov, S. Winitzki, Introduction to Quantum Effects in Gravity (Cambridge Univ. Press, Cambridge, 2007). https://cds. cern.ch/record/1122230

40. I.O. Starodub, Y. Zolotaryuk, Phys. Rev. B 90, 224509 (2014). https://doi.org/10.1103/PhysRevB.90.224509

41. G.W. Gibbons, S.W. Hawking, Phys. Rev. D 15, 2752 (1977a). https://doi.org/10.1103/PhysRevD.15.2752

42. G.W. Gibbons, S.W. Hawking, Phys. Rev. D 15, 2738 (1977b) https://doi.org/10.1103/PhysRevD.15.2738

43. L. Martina, O.K. Pashaev, G. Soliani, Phys. Rev. D 58, 084025 (1998). https://doi.org/10.1103/PhysRevD.58.084025

44. M.J. Ablowitz, D.J. Kaup, A.C. Newell, H. Segur, Phys. Rev. Lett. 31, 125 (1973a). https://doi.org/10.1103/PhysRevLett.31.125

45. M.J. Ablowitz, D.J. Kaup, A.C. Newell, H. Segur, Phys. Rev. Lett. 30, 1262 (1973b). https://doi.org/10.1103/PhysRevLett.30.1262

46. R. Schützhold, W.G. Unruh, Phys. Rev. Lett. 95, 031301 (2005). https://doi.org/10.1103/PhysRevLett.95.031301

47. M.P. Blencowe, V. Vitelli, Phys. Rev. A 62, 052104 (2000). https:// doi.org/10.1103/PhysRevA.62.052104

48. H. Wang, M. Hofheinz, M. Ansmann, R.C. Bialczak, E. Lucero, M. Neeley, A.D. O'Connell, D. Sank, J. Wenner, A.N. Cleland et al., Phys. Rev. Lett. 101, 240401 (2008). https://doi.org/10.1103/ PhysRevLett.101.240401

49. G. Romero, J.J. García-Ripoll, E. Solano, Phys. Rev. Lett. 102, 173602 (2009). https://doi.org/10.1103/PhysRevLett.102.173602

50. Y.-F. Chen, D. Hover, S. Sendelbach, L. Maurer, S.T. Merkel, E.J. Pritchett, F.K. Wilhelm, R. McDermott, Phys. Rev. Lett. 107, 217401 (2011). https://doi.org/10.1103/PhysRevLett.107.217401

51. O. Kyriienko, A.S. Sørensen, Phys. Rev. Lett. 117, 140503 (2016). https://doi.org/10.1103/PhysRevLett.117.140503

52. E. Martín-Martínez, L.J. Garay, J. León, Phys. Rev. D 82, 064028 (2010). https://doi.org/10.1103/PhysRevD.82.064028

53. X. Busch, R. Parentani, Phys. Rev. D 89, 105024 (2014). https:// doi.org/10.1103/PhysRevD.89.105024

54. J. Steinhauer, Phys. Rev. D 92, 024043 (2015). https://doi.org/10. 1103/PhysRevD.92.024043

55. J.R.M. de Nova, F. Sols, I. Zapata, N. J. Phys. 17, 105003 (2015). http://stacks.iop.org/1367-2630/17/i=10/a=105003

56. S. Robertson, F. Michel, R. Parentani, Phys. Rev. D 96, 045012 (2017). https://doi.org/10.1103/PhysRevD.96.045012

57. Z. Tian, S.-Y. Chä, U.R. Fischer, Phys. Rev. A 97, 063611 (2018). https://doi.org/10.1103/PhysRevA.97.063611

58. I. Kukuljan, S. Sotiriadis, G. Takacs, Phys. Rev. Lett. 121, 110402 (2018). https://doi.org/10.1103/PhysRevLett.121.110402

59. J. Gegenberg, G. Kunstatter, Phys. Rev. D 58, 124010 (1998). https://doi.org/10.1103/PhysRevD.58.124010

60. M. Cadoni, Phys. Rev. D 58, 104001 (1998). https://doi.org/10. 1103/PhysRevD.58.104001

61. A. Kundu, Phys. Rev. Lett. 99, 154101 (2007). https://doi.org/10. 1103/PhysRevLett.99.154101 